\title{
Field testing of a low-cost, self-referenced all-fibre polarimetric current sensor for the monitoring of current in the high-speed railway catenary \\ Massimo L. Filograno ${ }^{* a}$, Pedro Corredera ${ }^{\mathrm{a}}$, Miguel Gonzaléz-Herráez ${ }^{\mathrm{b}}$ \\ anstituto Óptica, CSIC, Madrid, Spain \\ ${ }^{\mathrm{b}}$ Departamento de Electrónica, UAH, Alcalá de Henares, Spain;
}

\begin{abstract}
In this paper we present the field testing results of a low-cost all-fibre polarimetric current sensor for the monitoring of current in the European high-speed railway network. The sensor fulfils the requirements of robustness, sensitivity, accuracy and cost required for the monitoring of catenary current in changeover sections. Changeover sections are nonfed sections of the catenary that are placed between sections fed with different phases and that introduce discontinuities in the current collection done by the train. Since the train passes at high-speed between the two sections, an electric arc can be formed that may lead to significant damage of the infrastructure. To avoid this situation, it is essential to ensure the switch-off of the current collection before arriving and switch-on again when the changeover section is passed. An adequate protection system that monitors the current in the catenary before the changeover section allows to trigger the necessary protection mechanisms in the infrastructure. Efficient, robust and lightweight electrical current sensors are therefore essential for this security system.

The sensor proposed here uses the Faraday magneto-optic Effect with a well-known polarimetric interrogation method. The optical configuration is extremely simplified through the use of few cost-effective, all-fibre devices with a simplified alignment. It allows high sensitivity for low current values, demonstrating a resolution below the ampere level with a dynamic range up to $500 \mathrm{~A}$.

The setup is electronically self-referenced to reduce the effect of small misalignments in the polarization, power variations in the optical source, temperature changes, birefringence effect and bending-induced attenuation in the lead fibre. A set of temperature tests in a climatic chamber were also performed in a range of temperature between -20 up to $80^{\circ} \mathrm{C}$, to assess the robustness of the device to operating temperature variations. The field tests include tests during commercial operations and special tests simulating fault conditions.
\end{abstract}

Keywords: optical fibre, current sensor, polarimetric sensor, Faraday Effect, high-speed railway, catenary

\section{INTRODUCTION}

The railway traffic in European networks is rapidly increasing during the last years, and is foreseen an increase of three times the present freight and passengers levels by 2020 [1]. Considering this growth, a number of $\mathrm{R}+\mathrm{D}$ initiatives have been taken in order to optimize the railway infrastructures maintenance and availability. These initiatives are especially important considering the important deployment of high-speed railway lines all over Europe.

A key issue in high-speed railway lines is the protection of the catenaries in changeover sections. Changeover sections are non-fed sections of the catenary that are placed between sections fed with different phases. These sections are located at intervals of several tens of kilometers and they introduce discontinuities in the current collection done by the train. Since the train passes at high-speed the electric arc formation at the end of the fed zone [2] can cause a strong wear [3] and short-circuits between the two fed sections. To avoid a strong arc formation, it is essential to ensure the correct operation of the train before and after the changeover section: the train must switch-off the current collection before arriving and switch-on again when the changeover section is passed. The non-fed section is essentially passed by inertia.

Whenever the train does not switch-off the current collection before arriving, electric arcs may form and destroy the railway catenary. To avoid these situations, it would be necessary to activate adequate protection systems at the

*massimo@io.cfmac.csic.es 
infrastructure level. These should imply monitoring the current in the catenary before the changeover section. Whenever a train passes without switching off the current collection, adequate signaling should be provided and the switching process should be done by the infrastructure itself. Thus, an exhaustive control of the electrical phase distribution in the changeover section must be performed. Efficient and robust electrical current sensors are therefore essential for their inclusion in the measurement and security systems of the railway networks. Essentially, the sensors developed for this application have to be very sensitive to low currents (to ensure detection of all the unwanted events) and only moderately precise for high currents.

Several approaches have been used to perform electrical current measurements in the railway catenary. Sensors based on Hall Effect have been mostly used so far, but they do not normally provide good isolation at $25 \mathrm{KV}$ and beyond (or they become extremely expensive). Modern high-speed railways run on these voltage levels, and thus optical current transformers are much more interesting for this application. Schemes for optical current transformers can be polarimetric [4] or interferometric [5,6], and there are also sensors based on magnetostrictive or piezoelectric transducers with Bragg gratings written in optical fibres [7,8].

In this work, we demonstrate a polarimetric current sensor based on the Faraday magneto-optic effect for the development of a cost-effective all-fibre current sensor for this application. Our setup is specially adapted to the requirements of this application, namely: AC current measurements with high sensitivity, dynamic range up to $500 \mathrm{~A}$, moderate accuracy over the full range, low cost and robustness to environmental and mechanical changes. The setup is electronically self-referenced to account for small misalignments in the polarization and power variations in the optical source.

\section{SENSOR SETUP}

Our current sensor makes use of the Faraday Effect. The Faraday Effect is a magneto-optic effect by which a linearly polarized light passing through a suitable material rotates an angle $(\theta)$ in proportion to the magnetic field parallel to the optical axis. This Faraday rotation angle is therefore proportional to the magnitude of the magnetic flux density $(B)$ and the propagation inside the optically active material. The proportionality constant is the well known Verdet constant $(V)$. Thus, the Faraday rotation in the most general case can be calculated with an integral along the optical path given by the equation (1)

$$
\theta(t)=\int V(\lambda, T) B(t) \cdot d l
$$

For a homogenous medium and in the presence of homogenous magnetic field and for a reasonably monochromatic light the Faraday rotation can be simplified in equation (2)

$$
\theta(t)=V B(t) L
$$

The magnetic field generated by a linear conductor is constant along paths parallel to the conductor, thus considering the proportionality between the current in a linear conductor and the magnetic field generated, joining all the constants in one, we can simplify our relation in the equation (3)

$$
\theta(t)=k I(t)
$$

The sensor arrangement is shown in figure 1, where the interrogation unit and the sensor head can be seen. The interrogation unit contains the light source, which is a super luminescent diode (SLD) providing relatively narrowband and almost linearly polarized beam. This beam is launched through an optical fibre isolator to a $3 \mathrm{~dB}$ coupler.

After the coupler, a passive depolarizer reduces considerably the polarization in the light that is injected in the lead fibre to reduce to the minimum the influence of this last in the polarization. The resulting interrogation unit is quite compact. It is meant to be installed in a control station relatively far from the sensing head. Once the system is adjusted, no further maintenance should be done. 


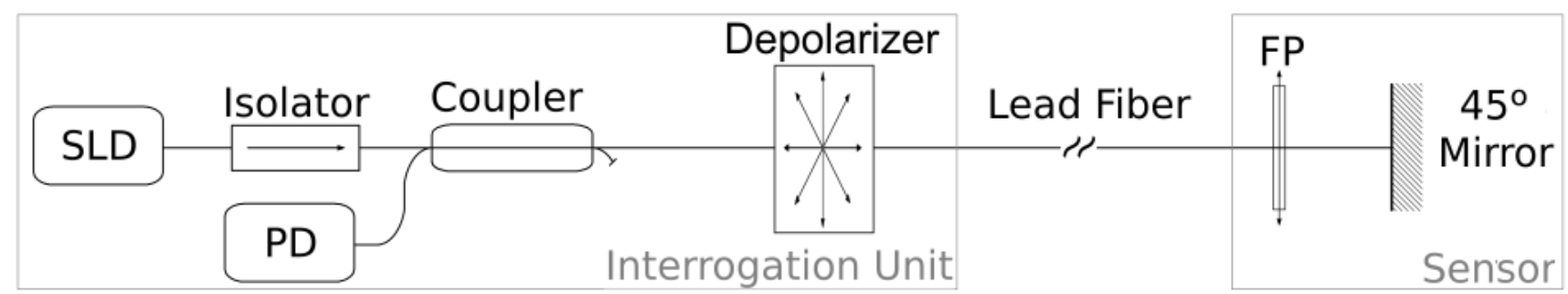

Figure 1. Scheme of the implemented polarimetric current sensor. Note that the packed sensor is a monolithic, low-weight block that can be easily installed in the catenary.

The sensor head is composed by two main elements: an in-fibre polarizer (FP), and a modified Faraday mirror. The power of the light in the output of the polarizer is maintained constant by the depolarizer. Any residual polarization sensitivity is accounted for in the electronic self-referencing scheme. The key point of the setup is this modified Faraday mirror. Essentially, this device is a based on a conventional low-cost and widely available Faraday mirror in which several permanent neodymium magnets have been added (figure 2).

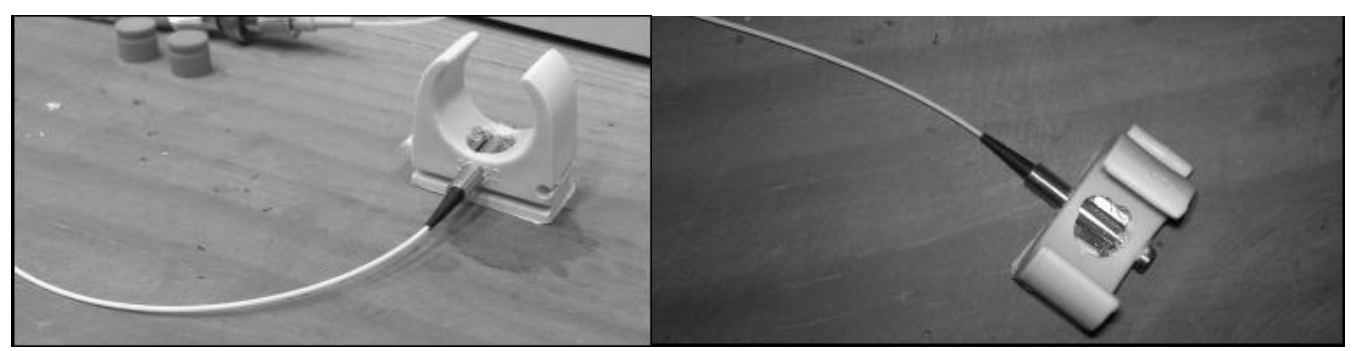

Figure 2. The modified Faraday mirror, packed with the additional neodymium magnets and a holder, ready to be directly adapted to the railway catenary.

The magnets allows a wide tuning of the angle of rotation introduced by the mirror, and therefore the working point in the transfer function of the sensor sketched in figure 3 .

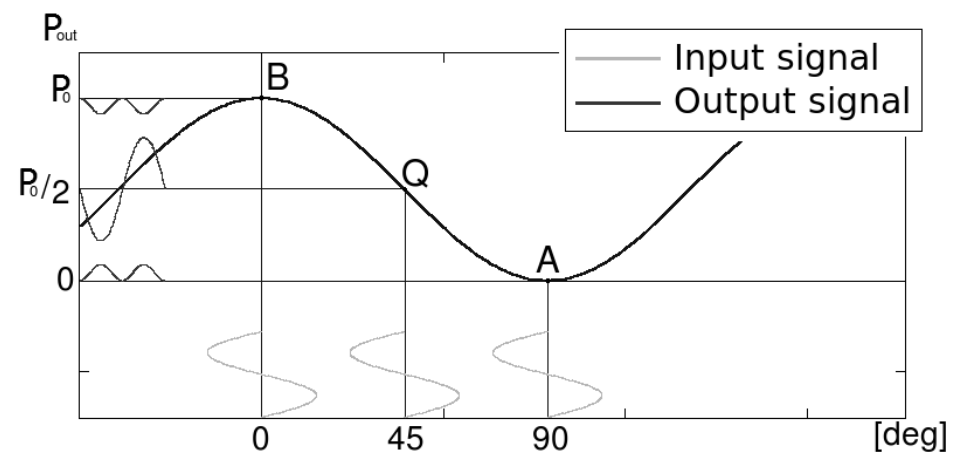

Figure 3. Plot of the optical power received by the PD as a function of the rotation angle introduced in the Modified Faraday Mirror.

When no modification of the original Faraday rotation $\left(90^{\circ}\right)$ is selected (point A of the transfer function in figure 3), the sensitivity to low currents is zero. Thus, this is an undesired working point. By shifting the position of the magnets one 
can tune the working point to introduce an additional rotation of $45^{\circ}$ (point $\mathrm{Q}$ in the transfer function), so that the final output can be written as

$$
P_{\text {out }}=P_{0} \cos ^{2}\left(k I_{c}+\pi / 4\right)
$$

where $P_{\text {out }}$ is the output optical power, $P_{0}$ is the input optical power, $I_{\mathrm{c}}$ is the current intensity, $k$ is a constant that take in count geometrical and physical parameters $\left(\mu_{0}\right.$ the vacuum magnetic permeability, the Verdet constant, the optical path length of the crystal, the geometry of the conductor and the position of the sensor respect the conductor under test). Q is the working point of maximum sensitivity. The alignment of the magnets is extremely simple: we introduce an AC current and we maximize the AC signal in the PD. With an additional DC current we can verify that we are in the midrange between two points of minimum sensitivity. Once the alignment is done, the magnets are glued and no further movement of the magnets is done.

In our setup, we can get a relatively good linearity in the range 0-500 A with a resolution well below the ampere level. For a maximum current value of 500A, the rotation angle due to the magneto-optic effect keeps in the approximately linear response region. Thus, the setup is considered linear over the whole range with a constant sensitivity. Nonlinearities below 10\% should be expected in the maximum current value. The reflective configuration we use duplicates the effective length of the material under magnetic field effect, and makes easier the installation of the sensor for field measurements (single cable, simplified alignment). This ensures a very high sensitivity in the setup, which is one of the requirements for this application. The Faraday mirror is spliced directly with the polarizer in a very short length of fibre to reduce to the minimum the effect of random birefringence in the fiber connection between the polarizer and the modified Faraday mirror.

Considering the purpose of the sensor we deliberately prefer a better sensitivity to small currents although losing accuracy at high currents. This is why the sensor is placed in close contact to the conductor under test. In this way also far fields of the other parts of the electric circuit become not relevant in the measurement, and their influence can be reduced further by calibration. It is possible to consider mismatches in sensitivity generated by the use of a Faraday mirror of a different manufacturer. In this scenario is important to consider a new calibration with the electric setup.

The complete system, including the electronic signal processing, has been designed to work with AC currents of $50 \mathrm{~Hz}$. This allows using electronic filters in detection to improve the signal to noise ratio. Modulation and demodulation of the optical power is also achieved to improve the signal to noise ratio by means of synchronous demodulation. The fact that the signal is AC also allows introducing a simple electronic self-referencing technique: an Automatic Gain Control. Thus, any small variation in the optical power of the source or the polarization controller alignment does not affect the setup since the gain is adapted so as to keep the mean value constant (both the mean value and the amplitude of the AC component depend on $P_{0}$ ).

\section{CALIBRATION AND TEMPERATURE TEST}

The sensor was tested in the lab with $50 \mathrm{~Hz} \mathrm{AC}$ current in the range of 0-700 [A]. The measured output remains stable for fixed values of current, regardless of movements, temperature variations (in the $15-25^{\circ} \mathrm{C}$ range) and power variation of the SLD. During the first part of the tests we found stability, a good S/N ratio and a useful immunity to changes of polarization and power in the SLD when the ACG-circuit is active. Movements in the lead fibre do not affect significantly the measured value, within the required accuracy for this application $(\sim 10 \%)$. The analog transfer function of the circuit is shown in figure 4. We can see a good sensitivity at low currents (we settled the gain of the last stage to have $\cong 10 \mathrm{mV} / \mathrm{A}$ at the output) and a slight sensitivity loss for higher currents. Typical train currents are normally below $400 \mathrm{~A}$. 


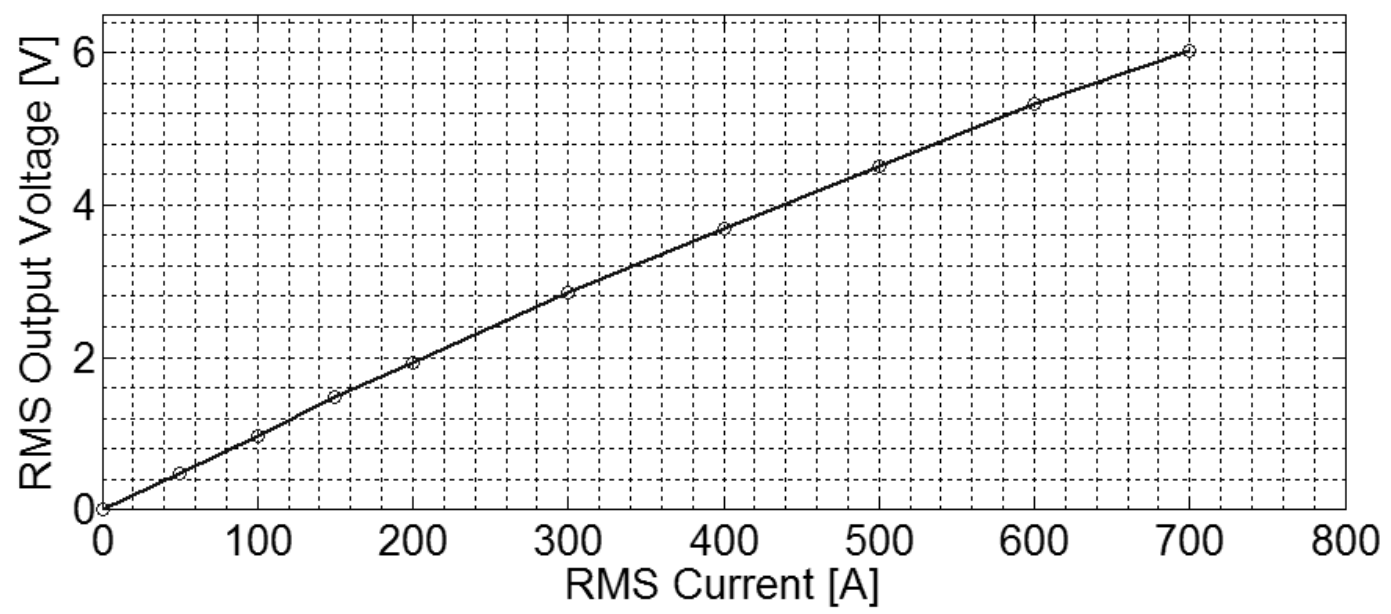

Figure 4. The output voltage of the complete sensor setup as a function of the RMS current fed through a test conductor with the same geometry of the catenary conductor. The current and its RMS value are provided by the LET-400-RDC current generator manufactured by EuroSMC.

In figure 5 we present the results of the temperature test in climatic chamber for the range $[-20,80]^{\circ} \mathrm{C}$. The cycle starts with the ambient temperature and it continue toward the maximum of about $80^{\circ} \mathrm{C}$ and conclude moving step by step toward the minimum of about $-20^{\circ} \mathrm{C}$. We proceeded with 7 steps with settle time of 20 minutes and a stabilizing time of about 20 minutes. The measure of the output voltage has been made for a current in the test conductor of 25 and 50A. Both in the calibration and in the temperature test the conductor under test have similar geometry of the conductor of the catenary.

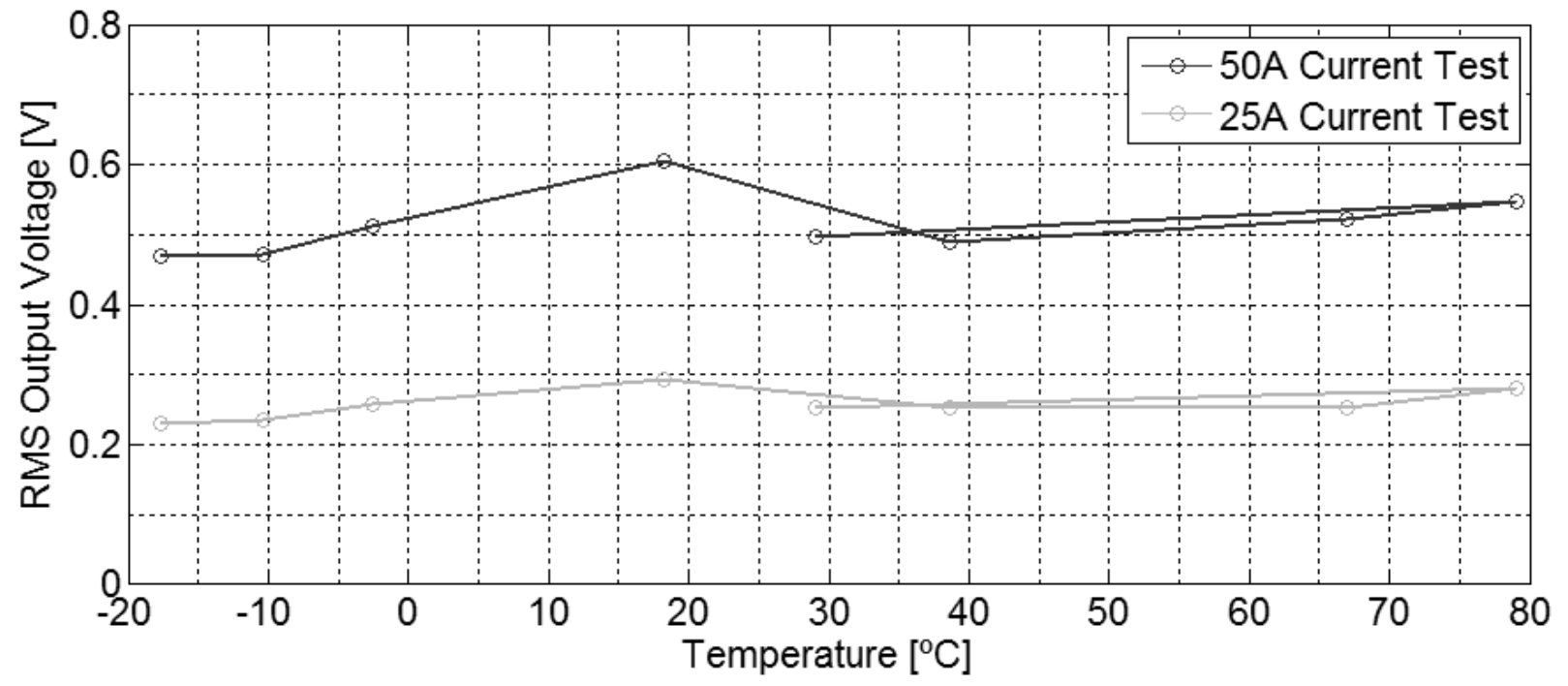

Figure 5. The output voltage during the temperature test in the climatic chamber. The test has been made for 25 and $50 \mathrm{~A}$ of current in the conductor under test.

From the analysis of the data of the temperature test we noticed a maximum variation in the measure of about $10 \%$. Even with this amount of error, the detection of current consumption by the train before the changeover section remains reliable. 


\section{EXPERIMENTAL RESULTS}

In figure 6 we see a photo of the installation, performed in Anchuelo, near Madrid, in the High Speed Line MadridBarcelona. The sensor is installed in the cable drop wire. The drop wires are vertical wires with mechanical and electrical function connected at regular distances between the catenary wire (the wire in the top that has a supporting function) and the contact wire (in electric contact with the pantograph).

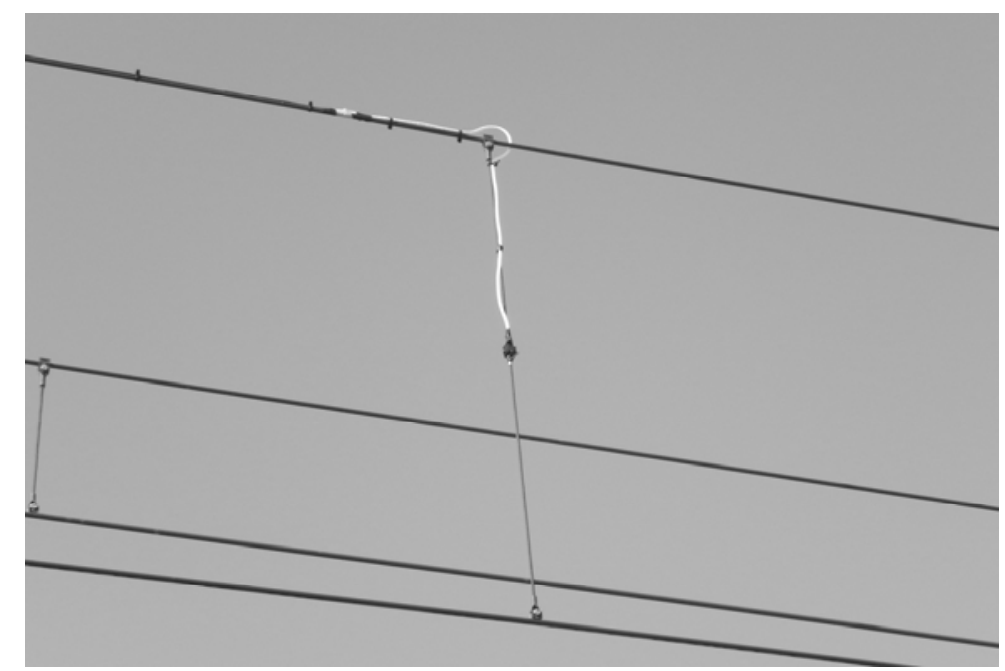

Figure 6. The site where the lightweight head sensor is mounted in the drop wire that joint the messenger wire with the contact wire of a high speed railway infrastructure.

When the train is passing just below the sensor, the maximum current flowing in the drop wire is generally in the order of a quarter part of the total current drained by the train. The sensor has been positioned in the zone before the changeover section where in normal condition the train must pass with the engine supply switched off. In this disposition the measure of a relevant current is related with a potentially dangerous condition that must be fronted with a shutdown of the power supply.

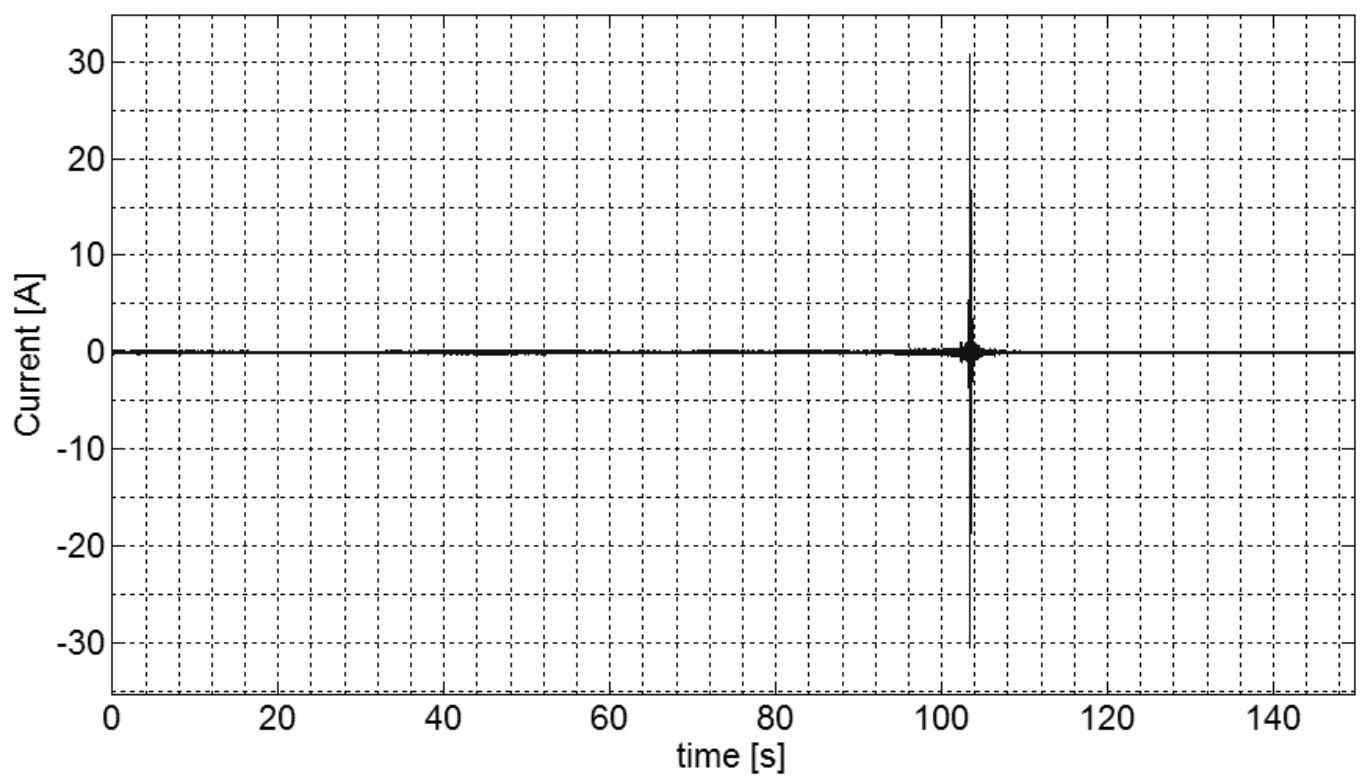

Figure 7. The RMS current measured by the sensor while the pass of the test train consuming $2.5 \mathrm{MW}$ of power. 
As expected for the geometry of the electric conductors, the train generated a brief peak of consumption in the measurement. In figure 7 we show the current measured for a test train consuming $2.5 \mathrm{MW}$. The power consumption of the test train is an average value for a typical train in commercial operation; therefore the expected maximum current, in fault condition, and for a commercial high speed train, is near the double. The acquisition is made with an analog to digital converter circuit (ADC) with a resolution of $12 \mathrm{bits}$ and a sampling rate of $1 \mathrm{kHz}$. The data was treated with a digital filter with the objective of reducing the noise and improve the resolution. A detail of the same acquisition is in figure 8 that shows the current's cycles in the conductor under test.

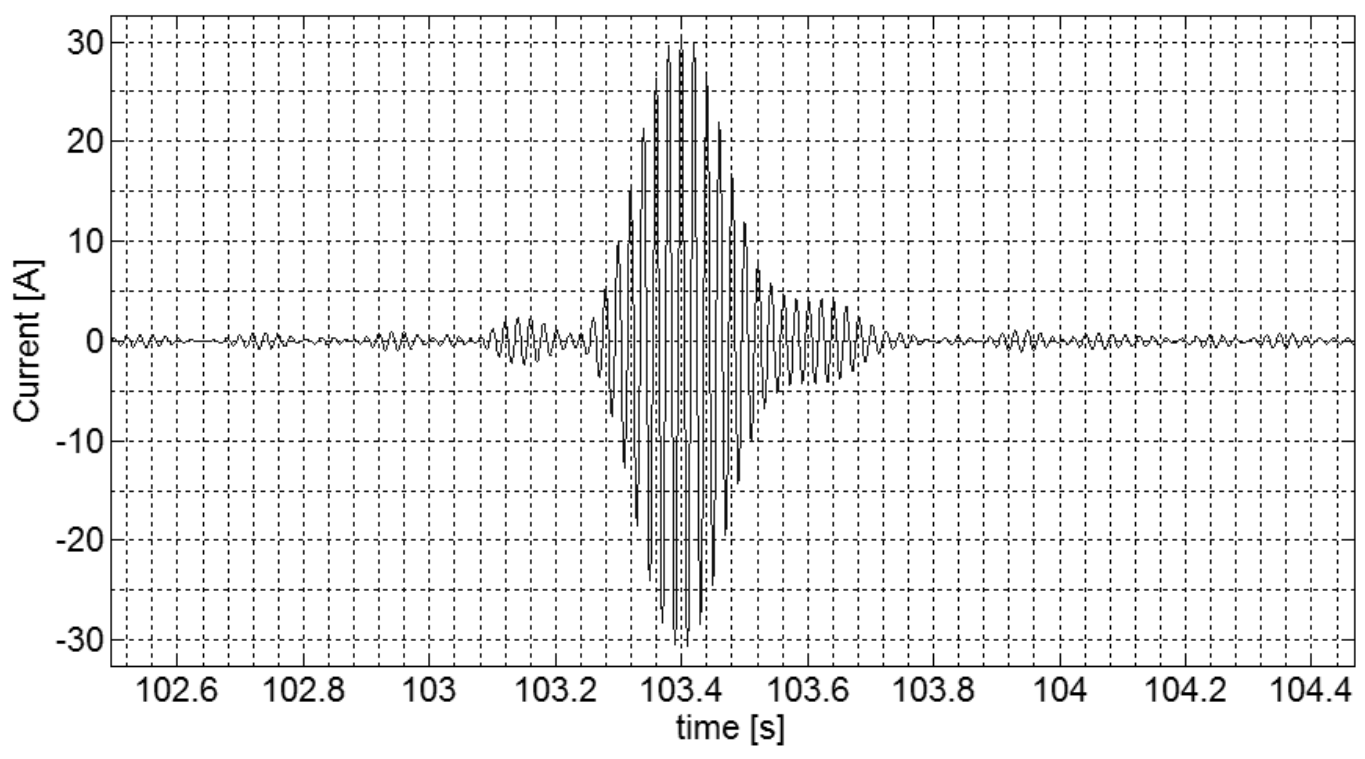

Figure 8. Detail of the current measured by the sensor while the pass of the test train consuming $2.5 \mathrm{MW}$ of power.

It is important to notice the reduced value of noise outside the peak, leading to a robust determination of current consumption in the ampere level.

\section{CONCLUSIONS}

We have described a low-cost all-fiber current sensor that fulfills the requirements of robustness, sensitivity, accuracy and cost required for the monitoring of catenary current in changeover sections of the high-speed railway network. Its optical configuration is simplified through the use of few devices with extremely simplified alignment. We reported the temperature test result that shows a moderate stability in sensitivity over a broad range of temperatures. Finally we reported the field tests that confirm the high sensitivity for low current values with a resolution below the ampere level. The accuracy is comparably low but sufficient for the application.

\section{AKNOWLEDGEMENTS}

This work has been partially supported by Ministerio de Fomento (Spain) projects MIFFO (Monitorización de Estructuras Ferroviarias empleando Tecnologías de Fibra Óptica), ref. PEIT 77/07, and IFZONE (Investigación de técnicas avanzadas para la explotación ferroviaria en las Zonas neutras de catenaria de las líneas de Alta Velocidad), ref. P 39/08, and Comunidad de Madrid Project FACTOTEM II (Fotónica aplicada a la creación de tecnologías ópticas y su transferencia a empresas Madrileñas-II), ref. S2009/ESP-1781 


\section{REFERENCES}

[1] G. Laffont, N. Roussel, S. Rougeault, J. Boussoir, L. Maurin, P. Ferdinand, "Innovative FBG sensing techniques for the railway industry: Application to Overhead Contact Line Monitoring", in $20^{\text {th }}$ International Conference on Optical Fiber Sensors OFS'20, J. Jones et al. Ed., SPIE 7503, 2009

[2] B. Y. Lee, J. C. Kim, S. H. Han, S. G. Lee, "A Study on the arc induced by contact loss between pantograph and catenary ," autumn conference of Korean institute of Illuminating and electrical installation engineers, 2005.

[3] Giuseppe Bucca, Andrea Collina, "A procedure for the wear prediction of collector strip and contact wire in pantograph-catenary system", Wear 266 (2009) 46-59

[4] D. Alasia, L. Thévenaz, "A novell all-fibre configuration for a flexible polarimetric current sensor", Meas. Sci. Techn. 15, 2004

[5] K. Bohnert, P. Gabus, J. Nehring, H. Brändle and M. G. Brunzel "Fiber-optic current sensor for electrowinning of metals" Journal of Lightwave Technology 25(11), 2007

[6] F. Briffod, 2003 "Structures innovantes pour capteurs optiques de courants électriques" PhD Thesis Swiss Federal Institut of Technology, Lausanne

[7] J. Mora, A. Díez, J.L. Cruz, M.V. Andrés, "A magnetostrictive sensor interrogated by fiber gratings for DC-current and temperature discrimination", IEEE Photon. Technol. Lett. 12(12), 2000

[8] G. Fusiek, P. Niewczas, J.R. McDonald, "Feasibility study of the application of optical voltage and current sensors and an arrayed waveguide grating for aero-electrical systems", Sens. \& Act. A 147, 2008 